\title{
The effect of walls on a spatially growing supersonic shear layer
}

\author{
Mei Zhuang, ${ }^{\text {a) }}$ Paul E. Dimotakis, ${ }^{\text {b) }}$ and Toshi Kubotac) \\ Graduate Aeronautical Laboratories, California Institute of Technology, Pasadena, California 91125
}

(Received 9 August 1989; accepted 22 December 1989)

\begin{abstract}
The inviscid instability, with respect to supersonic disturbances of a spatially growing plane mixing layer inside parallel flow guide walls, is investigated using linear stability analysis. For supersonic convective Mach numbers, it is found that the maximum amplification rates of the shear layers approach an asymptotic value and that this maximum amplification rate increases to its maximum value and decreases again as the distance between the walls decreases continuously. Contour plots of the pressure perturbation fields indicate that there are waves propagating outward from the shear layer along the Mach angle, and that the walls provide a feedback mechanism between the growing shear layer and this compression/expansion wave system. The streak lines of the flow confirm that the spreading rate of the shear layer is unusually small for supersonic disturbances.
\end{abstract}

\section{INTRODUCTION}

Lees and $\operatorname{Lin}^{1}$ gave the general description of linear stability characteristics of compressible shear layers. The numerical results of Lessen et al. ${ }^{2}$ showed that the flow is generally unstable with respect to supersonic disturbances, although the amplification rate is smaller than for subsonic disturbances. Experimental results of turbulent shear layer mixing by Papamoschou and Roshko $0^{3,4}$ indicated that the normalized growth rate (unity for incompressible flow) reaches an asymptotic value for supersonic convective Mach numbers. The same qualitative behavior was found by Bogdanoff ${ }^{5}$ in his analysis of several previous experimental investigations of supersonic shear layers. Recently, Ragab and $\mathrm{Wu}^{6}$ and Zhuang et $a l^{7}$ studied the effects of shear layer Mach number, temperature ratio, velocity ratio, and temperature profile on the stability characteristics of two-dimensional (2-D) unbounded free shear layers with 2-D spatially growing disturbances. They found that there is a nearly universal dependence of the normalized maximum amplification rate on the convective Mach number, and that this amplification rate decreases continuously as the convective Mach number increases. These stability analyses, however, were carried out for unbounded flow, as opposed to the experiments that were conducted in enclosed test sections. Sandham and Reynolds ${ }^{8}$ investigated the compressible shear layers using both linear theory and direct simulation. They showed that linear theory can be very useful in understanding the physics of free shear layers and the growth rate of the developed plane mixing layer.

The study of the compressible boundary-layer stability theory carried out by Mack $^{9,10}$ indicated that as long as there is a region of supersonic relative flow there will always be unstable modes present in the boundary layer. For a shear layer inside a rectangular channel, Tam and $\mathrm{Hu}^{\text {" }}$ showed that the coupling between the motion of the shear layer and the acoustic modes of the channel produces new instability

\footnotetext{
a) Graduate student, Aeronautics.

b) Professor, Aeronautics and Applied Physics.

c) Professor, Aeronautics.
}

waves for the spatially growing mixing layers. Greenough $e t$ $a l .^{12}$ considered the effects of the walls on a compressible confined temporal mixing layer. They showed that there were two general types of instabilities: confined KelvinHelmholtz modes and supersonic wall modes.

In this paper, we have studied the linear instability of a spatially growing plane shear layer inside parallel walls. The shear layer flow considered is inviscid and formed by the same gases in the two streams. The mean flow is treated as parallel. The purpose of this paper is to give a description of how the instability characteristics of the shear layer are affected by the flow guide walls and by the distance between the walls. For supersonic disturbances, the reflections of the compression/expansion waves from the walls can be seen in contour plots of the pressure perturbation fields. This feedback mechanism provided by the walls between the spatially growing supersonic shear layer and the wave system makes the flow more unstable than the corresponding free supersonic shear flow, provided the walls are not too close to the layer. Also, the flow patterns of the shear layers are obtained by calculating the streak lines of the shear layers.

\section{FORMULATION}

Consider a two-dimensional parallel flow of two streams. With upper stream quantities as the reference and the local layer thickness as the length scale, the general dimensionless quantity of flow field can be expressed as

$$
Q(x, y, t)=\bar{Q}(y)+Q^{\prime}(x, y, t),
$$

where $\bar{Q}(y)$ is the mean flow quantity. Assume that the flow is subjected to small disturbances propagating in the $x$ direction having the form

$$
Q^{\prime}(x, y, t)=q(y) \exp [i \alpha(x-c t)],
$$

where $\alpha$ is a dimensionless complex wavenumber and $c$ is a dimensionless complex wave velocity.

From the basic disturbance equations for infinite Reynolds number, the second-order differential equation for the dimensionless pressure disturbances can be derived, ${ }^{7}$ i.e., 


$$
\begin{aligned}
\pi^{\prime \prime}(y) & -\left(\frac{2 \bar{U}^{\prime}}{\bar{U}-c}-\frac{\bar{T}^{\prime}}{\bar{T}}\right) \pi^{\prime}(y) \\
& -\alpha^{2}\left(1-\frac{M_{1}^{2}}{\bar{T}}(\bar{U}-c)^{2}\right) \pi(y)=0,
\end{aligned}
$$

where $M_{1}$ is the upper stream Mach number and $\bar{U}$ and $\bar{T}$ are, respectively, the dimensionless mean velocity and temperature profiles described by a hyperbolic tangent profile and by the Crocco-Busemann relation. ${ }^{7}$ The general configuration of the shear layer inside parallel walls is shown in Fig. 1. The dimensionless shear layer thickness, $\delta$, is defined in a boundary-layer sense, i.e.,

$$
\left|\left[U_{1}-\bar{U}(\delta)\right] / U_{1}\right|<\epsilon, \quad\left|\left[\bar{U}(-\delta)-U_{2}\right] / U_{2}\right|<\epsilon,
$$

where $\epsilon$ is taken as $0.002 ; 2 h$ is the dimensionless distance between the walls.

In the regions of $\delta<y<h$ and $-h<y<-\delta$, the flow is effectively uniform, i.e., the mean velocity and temperature are constants, and Eq. (3) becomes

$$
\pi^{\prime \prime}-\lambda_{k}^{2} \pi=0
$$

with

$$
\lambda_{k}^{2}=\alpha^{2}\left[1-\left(M_{1}^{2} / \bar{T}_{k}\right)\left(\bar{U}_{k}-c\right)^{2}\right],
$$

for $k=1,2$. The boundary conditions at the walls, i.e., $\pi^{\prime}(h)=\pi^{\prime}(-h)=0$, then yield the analytic solutions in regions 1 and 2 as

$$
\pi=A \cosh \left[\lambda_{1}(h-y)\right], \quad \pi=B \cosh \left[\lambda_{2}(h+y)\right],
$$

where $\lambda_{1}$ and $\lambda_{2}$ are given by Eq. (6), and $A$ and $B$ are constants to be determined by matching to the inner flow region.

To find the solution inside the shear layer, we use the analytic solution in region 2 as a starting solution and integrate $\mathrm{Eq}$. (3) numerically from $y=-\delta$ to $y=\delta$. The correct $\alpha$ is obtained, for a given $\omega=\alpha c$, by matching the numerical solution to the analytic solution at $y=\delta$ (shooting method). The Runge-Kutta method is used to integrate the equation and solve this eigenvalue problem.

The procedure for the calculations of the streak lines is the same as that used by Michalke. ${ }^{13}$ The motion of a particle is given by

$$
\frac{d x}{d t}=\epsilon_{1} u^{\prime}(x, y, t)+\bar{U}(y),
$$

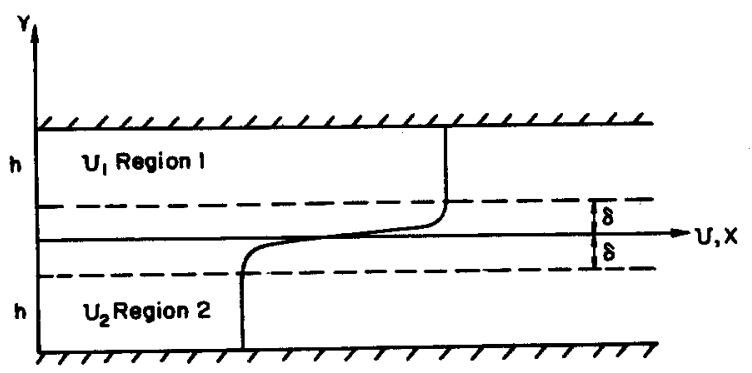

FIG. 1. The general configuration of a shear layer inside parallel walls.

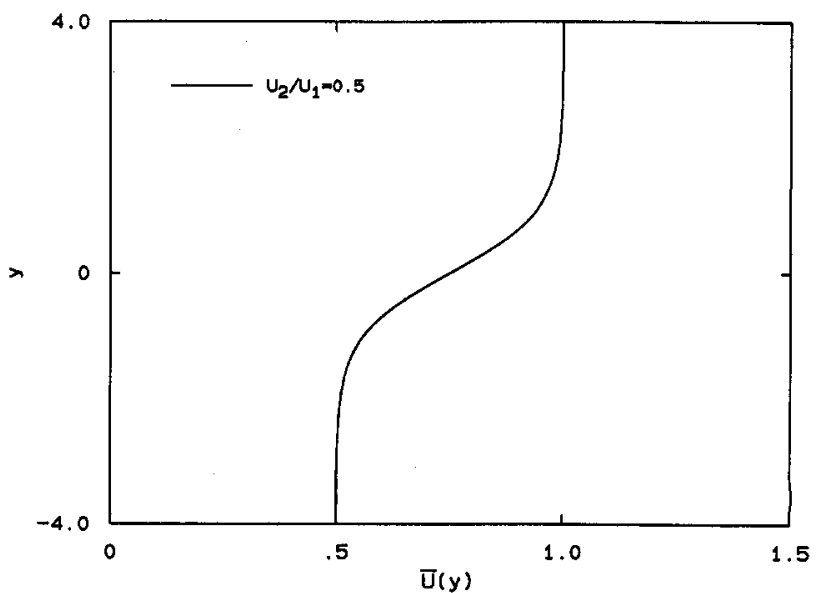

FIG. 2. Hyperbolic tangent dimensionless mean velocity profile for the velocity ratio $U_{2} / U_{1}=0.5$.

$$
\frac{d y}{d t}=\epsilon_{1} v^{\prime}(x, y, t) \text {. }
$$

With appropriate initial conditions, $x\left(t_{0}\right)=x_{0}, y\left(t_{0}\right)=y_{0}$, a path line can be determined. In order to plot each streak line for a fixed time, the path lines for various initial times, $t_{0}$, are calculated. In the calculations we have chosen $x_{0}=0$, $\epsilon_{1}=0.0005$ for subsonic convective Mach numbers, and $x_{0}=0, \epsilon=0.1$ for supersonic convective Mach numbers.

\section{RESULTS AND DISCUSSION}

The instability characteristics of shear layers with velocity and temperature profiles shown in Figs. 2 and 3 are determined for 2-D spatially growing disturbances with $h=12$ unit lengths at different Mach numbers $M_{1}$. The main result, shown in Fig. 4, is that when the convective Mach number of the flow is supersonic, as was also noted by Mack ${ }^{9,10}$ in his analysis of supersonic boundary-layer stability, there are

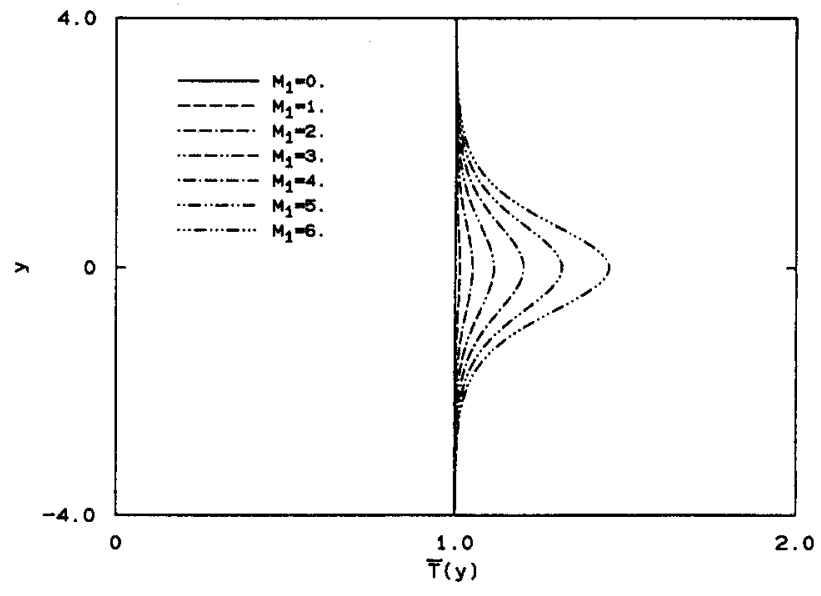

FIG. 3. Crocco-Busemann dimensionless mean temperature profiles for the case $U_{2} / U_{1}=0.5$ and $T_{2} / T_{1}=1.0$. 


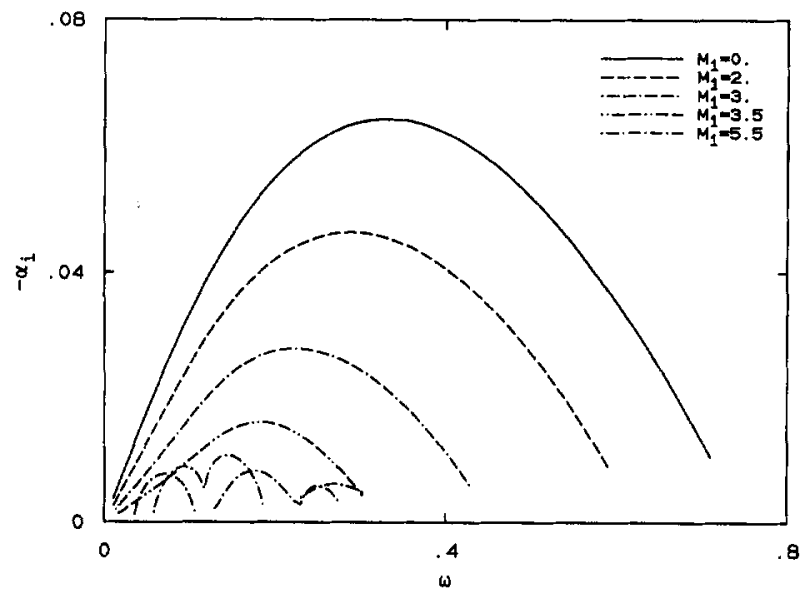

FIG. 4. Instability characteristics of the mean profiles in Figs. 2 and 3 at $h=12$ unit lengths and $\delta=3$ unit lengths.

many supersonic unstable modes, as opposed to only one for the case of subsonic convective Mach number. The existence of many supersonic instability modes in the case of shear layers has been known from the work of Tam and $\mathrm{Hu} .^{11}$ Their results, however, were based on the instability calculations inside a three-dimensional rectangular channel. Figure 5 (the normalized maximum amplification rate versus convective Mach number $M_{c 1}$ ) indicates that the growth rate of the most unstable supersonic instability mode of shear layer with 2-D spatially growing disturbances approaches an asymptotic value as the convective Mach number becomes supersonic, which is in accord with the previously mentioned growth rate experiments. ${ }^{4}$ The convective Mach number here is defined for each stream as ${ }^{7}$

$$
M_{c l}=\left(U_{1}-c_{p}\right) / a_{1}, \quad M_{c 2}=\left(c_{p}-U_{2}\right) / a_{2},
$$

where $U_{1}, U_{2}$ and $a_{1}, a_{2}$ are the free-stream velocities and speeds of sound, respectively, and $c_{p}$ is the phase velocity of

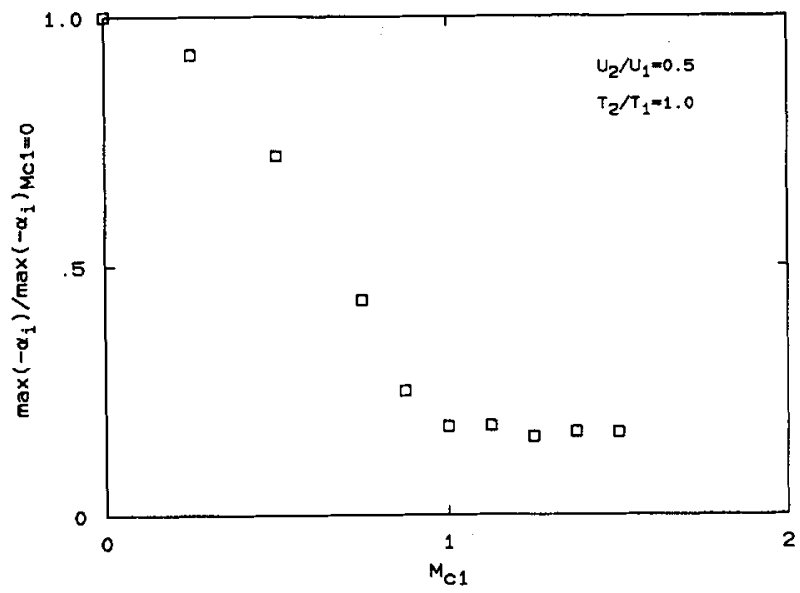

FIG. 5. Normalized maximum amplification rate versus $M_{c \mathrm{c}}$ at $h=12$ unit lengths and $\delta=3$ unit lengths.

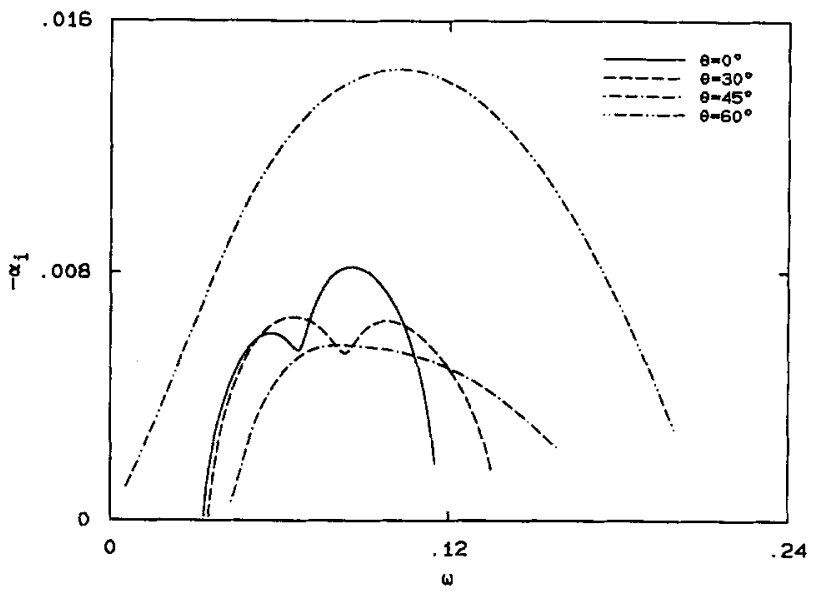

FIG. 6. Instability characteristics for 3-D spatially growing disturbances of the mean profiles at $\delta=3$ unit lengths, $h=20$ unit lengths, and $M_{1}=5.5$.

the disturbances. Alternative definitions of the convective Mach number have also been used ${ }^{3,4,6,8}$ in which a different estimate of the convective velocity of the large structures $U_{c}$ is used instead of $c_{p}$. For a 2-D mixing layer, comprised of free-stream fluids with the same ratio of specific heats, $U_{c}$ is estimated by

$$
U_{c}=\left(a_{1} U_{2}+a_{2} U_{1}\right) /\left(a_{1}+a_{2}\right) \text {. }
$$

In our calculations, $c_{p}$ is chosen to be the phase velocity of the most unstable eigenvalue. We think the definition given by Eq. (10) is more appropriate since the phase velocity of disturbances is derivable from the computations.

If a shear layer inside the parallel flow guide walls is subjected to a small 3-D spatially growing disturbance having the form

$$
Q^{\prime}(x, y, z, t)=q(y) \exp [i(\alpha x+\beta z-\omega t)],
$$

where $\beta$ is a dimensionless complex wavenumber in the $z$ direction and $\omega$ is a dimensionless real frequency, then the growth rate of the 3-D mode is larger than the corresponding 2-D mode at high convective Mach numbers over some range of propagation direction (see Fig. 6). This result shows behavior similar to the result of the previous studies of a free shear layer with 3-D spatially growing disturbances by Gropengiesser, ${ }^{14}$ Ragab and $\mathrm{Wu},{ }^{6}$ Jackson and Grosch, ${ }^{15}$ and Sandham and Reynolds. ${ }^{16}$ For a shear layer inside a rectangular channel, Tam and $\mathrm{Hu}^{17}$ considered 3-D spatially growing disturbances of the form

$$
\begin{aligned}
p^{\prime}(x, y, z, t)= & \bar{p}(y) \exp [i(\alpha x-\omega t)] \\
& \times \cos (2 m \pi z / B) \quad(m=0,1,2, \ldots),
\end{aligned}
$$

where $p^{\prime}$ is the perturbation pressure and $B$ is the breadth of the rectangular channel. They showed that 2-D supersonic instability waves have larger spatial growth rates than their 3-D counterparts for reasonably thick shear layers. We have conducted similar calculations of this problem, which are in agreement with the results of Tam and $\mathrm{Hu}$. The instability characteristics of 3-D modes have a similar behavior as the 


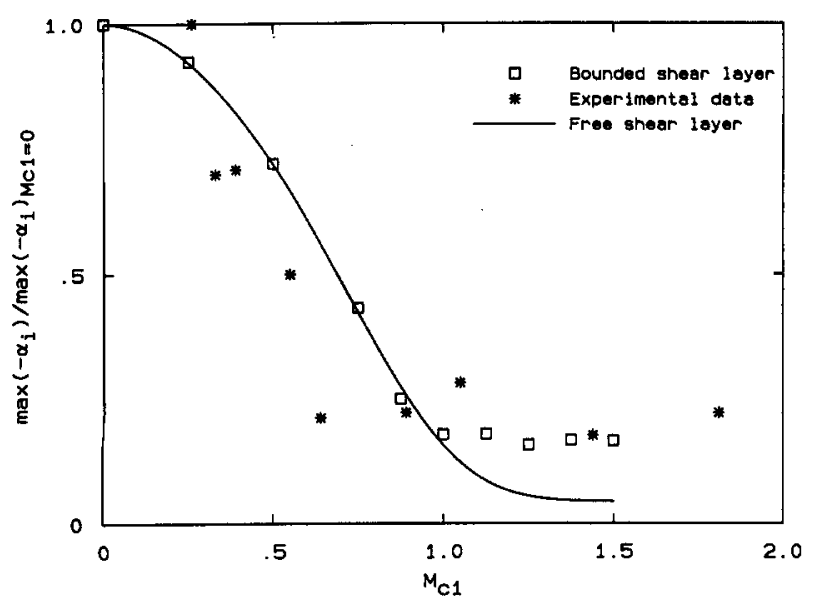

FIG. 7. A comparison of our data for a 2-D bounded shear layer with the experimental data of Papamoschou and Roshko and the function $F\left(M_{c 1}\right)$, which was obtained by least-squares fitting the data we obtained from the previous calculations of the 2-D free shear layers. ${ }^{7}$

2-D modes. As a consequence, 2-D modes were considered mainly in the work described below.

Comparisons of our results shown in Fig. 5 with the experimental results of Papamoschou and Roshko, ${ }^{4}$ and with the results obtained from the 2-D free shear layer calculations, ${ }^{7}$ are made in Fig. 7. We can see how walls affect the instability of the shear layer. The existence of the walls makes the shear layer more unstable and keeps the maximum amplification rates from reaching an asymptotically small value for supersonic convective Mach numbers, but has no discernible effect on shear layers with subsonic convective Mach numbers.

For a fixed upper stream Mach number $M_{1}$, with the same mean velocity and temperature profiles given as described above, the instability characteristics of the shear layers with 2-D spatially growing waves are calculated at different values of $h$ ( $h=20,16,12$, and 8 unit lengths). The most

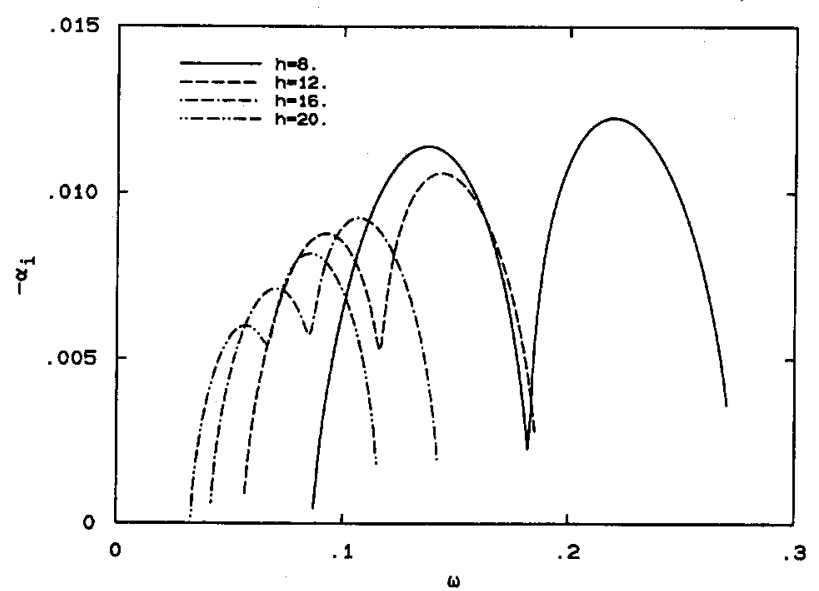

FIG. 8. Instability characteristics of the most unstable modes of the mean profiles for different values of $h$ at $\delta=3$ unit lengths and $M_{1}=5.5$. unstable supersonic instability mode, which is not the Kelvin-Helmholtz mode, moves to higher frequencies and its maximum amplification rate increases as the distances between the two walls decreases (see Fig. 8). But this maximum amplification rate reaches its maximum value and decreases as the distance between the walls decreases continuously (see Fig. 9). The instability characteristics of subsonic shear layers are not affected by the distance between the walls. We chose the thickness of the shear layer as the characteristic length in our calculations, which made the solution of a bounded shear layer with $h \rightarrow \infty$ approach that of the corresponding free shear layer. If we consider the distance between walls as the characteristic length, the growth rate of the supersonic instability waves scaled to this characteristic length decreases at a fixed frequency as the shear layer becomes thicker and thicker. ${ }^{17}$

Contour plots of the pressure perturbation fields, which combine the periodic term and the growth term, are shown in Figs. 10-13. Note that the convective Mach numbers of the shear layers corresponding to these contour plots are either close to, or over, unity, so the growth of the shear layers is small within the extent plotted. Figure 10 shows the flow with the same supersonic convective Mach numbers at both sides of the boundaries $\left(M_{c 1}=M_{c 2}=1.375\right)$. We can see that the compression/expansion waves propagate along the Mach angle $\mu=\arcsin \left(1 / M_{c 1}\right)$ or $\mu=\arcsin \left(1 / M_{c 2}\right)$ for supersonic convective Mach numbers. By measuring the Mach angle $\left(\mu=45.71^{\circ}\right)$, we estimate a convective Mach number of $M_{c}=1.397$, in close agreement with $M_{c 1}$ and $M_{c 2}$ estimated using Eq. (10). The variations in the strength of these compression/expansion waves suggest that these waves are reflected by the walls. Figure 11 is the contour plot for the free shear layer with the upper stream supersonic and lower stream subsonic $\left(M_{c 1}=1.81\right.$ and $\left.M_{c 2}=0.94\right)$. The upper flow compression/expansion waves propagate with the Mach angle $\mu=34.23^{\circ}$, i.e., $M_{c 1}=1.78$, but no reflections exist. From these two contour plots we can see that for supersonic disturbances, because of the existence of the walls, the energy carried by the wave system is reflected back to the shear layer instead of being radiated to the far field.

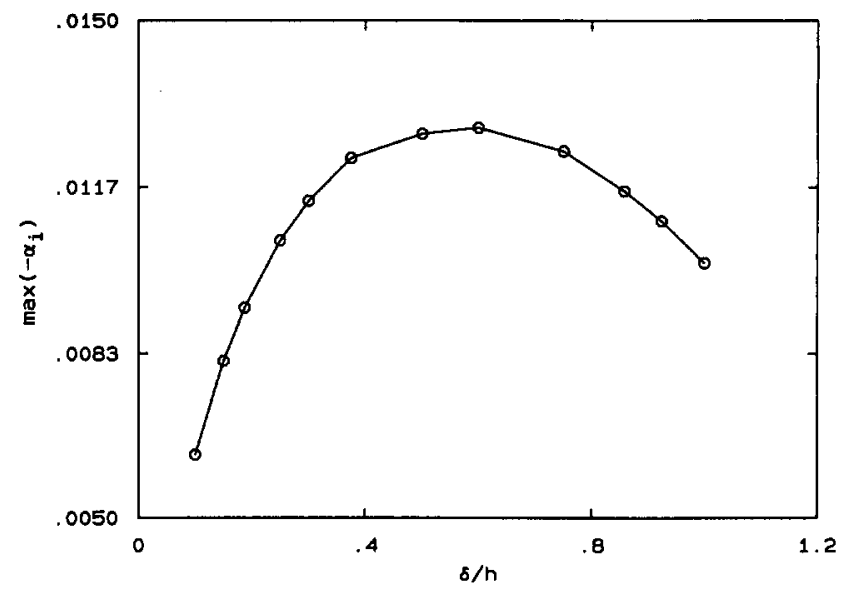

FIG. 9. The maximum amplification rate of supersonic instability mode versus $\delta / h$ at $\delta=3$ unit lengths and $M_{1}=5.5$. 


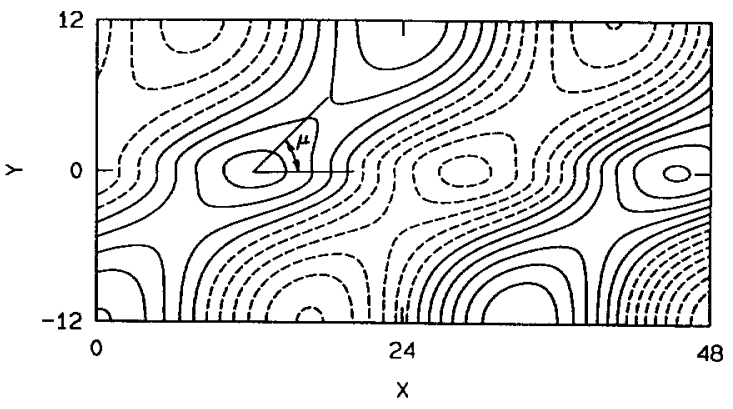

FIG. 10. Pressure pertrubation field of the most unstable mode for $\delta=3$ unit lengths, $h=12$ unit lengths, and $M_{1}=5.5$.

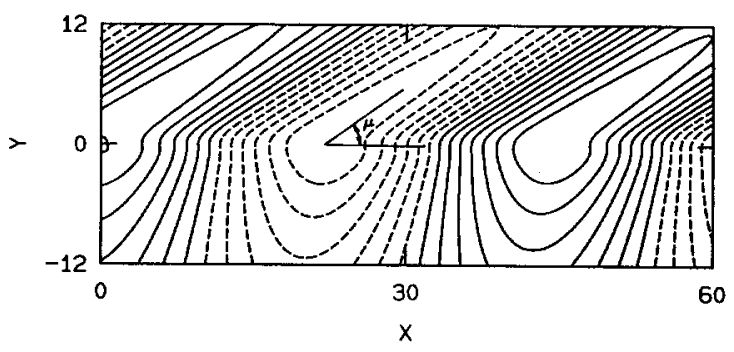

FIG. 11. Pressure perturbation field of the most unstable mode for the free shear layer at $M_{1}=5.5$.

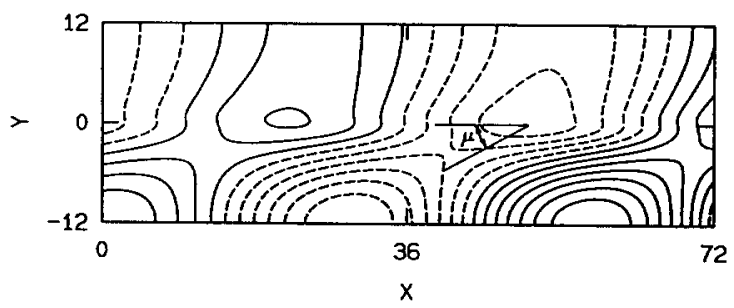

FIG. 12. Pressure perturbation field of the second unstable mode for $\delta=3$ unit lengths, $h=12$ unit lengths, and $M_{1}=5.5$.

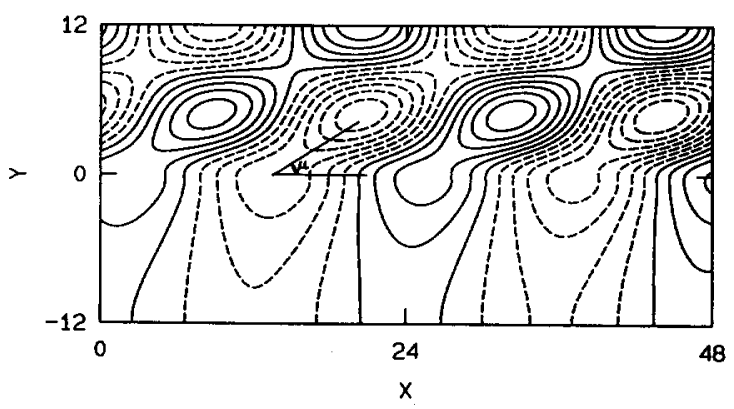

FIG. 13. Pressure perturbation field of the third unstable mode for $\delta=3$ unit lengths, $h=12$ unit lengths, and $M_{1}=5.5$.

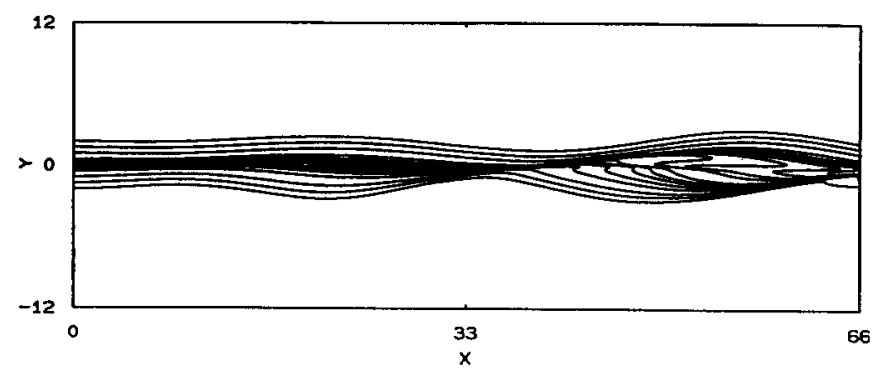

FIG. 14. Streak lines for the case $U_{2} / U_{1}=0.5$ and $T_{2} / T_{1}=1.0$ at $\delta=3$ unit lengths, $h=12$ unit lengths, and $M_{1}=5.5$ (supersonic convective Mach numbers $M_{\mathrm{r} 1}=M_{c 2}=1.375$ ).

The feedback mechanism between the growing supersonic shear layer and the wave system makes the bounded supersonic shear layer more unstable than the corresponding free supersonic shear layer, which is losing energy to acoustic radiation to the far field. ${ }^{10,18}$ Figures 12 and 13 are two other examples of contour plots for bounded shear layers; one is with the lower stream supersonic and upper stream subsonic, and the other is vice versa. Reflections of the compression/expansion wave system propagating along Mach angles for supersonic convective Mach numbers can also be seen in those figures.

In order to describe the flow patterns of the shear layers, streak lines were also calculated for the cases of maximum amplification rates. The results plotted in Figs. 14 and 15 represent streak lines corresponding to supersonic and subsonic disturbances, respectively. These flow patterns show that, for supersonic disturbances, the growth of the shear layer, as labeled by the streak lines, is much slower than that for subsonic disturbances, and the spreading rate of the shear layer is small. The existence of the compression/expansion waves in the mixing layer with supersonic disturbances may

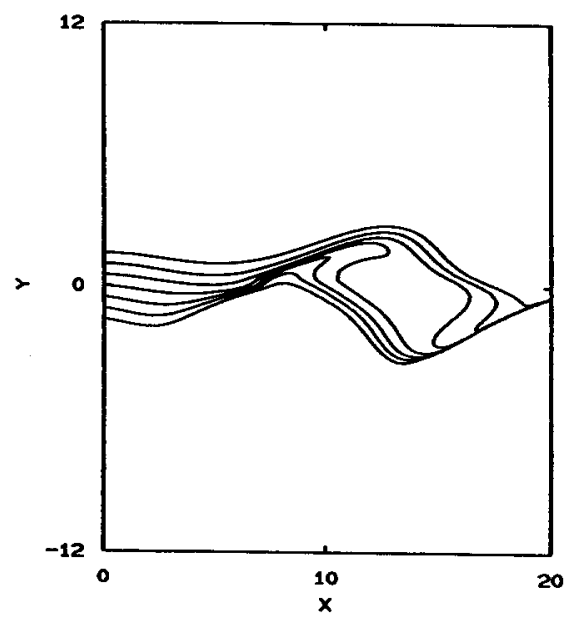

FIG. 15. Streak lines for the case $U_{2} / U_{1}=0.5$ and $T_{2} / T_{1}=1.0$ at $\delta=3$ unit lengths, $h=12$ unit lengths, and $M_{1}=2.0$ (subsonic convective Mach numbers $M_{c 1}=M_{c 2}=0.5$ ). 
cause the pressure gradients across the layer. Therefore the layer may be pinched by the pressure equalization.

\section{CONCLUSIONS}

Spatially growing plane mixing layers inside parallel guide walls were studied using linear instability analysis. The effects of the parallel walls and the distance between them on the instability characteristics of the shear layers were investigated. The distance between such walls is found to affect the amplification rates of the characteristics of the shear layers for supersonic convective Mach numbers. As the distance decreases continuously, the maximum amplification rate of the shear layer increases to its maximum value and decreases again. For supersonic convective Mach numbers, the reflections of the compression/expansion waves caused by the parallel walls provide a feedback mechanism between the growing shear layer structures and the wave system, so the bounded shear layers are more unstable than the corresponding free shear layers. The maximum amplification rates of the bounded shear layers are found to approach an asymptotic value as the convective Mach number is increased.

\section{ACKNOWLEDGMENT}

This research was supported by the Air Force Office of Scientific Research, Grant No. AFOSR-88-0155.

${ }^{1}$ L. Lees and C. C. Lin, NACA Tech. Note No. 1115, 1946.

${ }^{2}$ M. Lessen, J. A. Fox, and H. M. Zien, J. Fluid Mech. 25, 737 (1966).

${ }^{3}$ D. Papamoschou and A. Roshko, AIAA Paper No. 88-0162, 1986.

${ }^{4}$ D. Papamoschou and A. Roshko, J. Fluid Mech. 197, 453 (1988).

${ }^{5}$ D. W. Bogdanoff, AIAA J. 21, 926 ( 1983).

'S. A. Ragab and J. L. Wu, AIAA Paper No. 88-0038, 1988.

${ }^{7}$ M. Zhuang, T. Kubota, and P. E. Dimotakis, AIAA Paper No. 88-3538$\mathrm{CP}, 1988$.

${ }^{8}$ N. Sandham and W. Reynolds, AIAA Paper No. 89-0371, 1989.

${ }^{9}$ L. M. Mack, Jet Propulsion Laboratory, Document No. 900-277, Revision A, 1969.

${ }^{10}$ L. M. Mack, AGARD Report No. 709, 1984.

"C. K. W. Tam and F. Q. Hu, AIAA Paper No. 88-3675-CP, 1988.

${ }^{12} \mathrm{~J}$. Greenough, J. Riley, M. Soetrisno, and D. Eberhardt, AIAA Paper No. 89-0372, 1989.

${ }^{13}$ A. Michalke, J. Fluid Mech. 23, 521 (1965).

${ }^{14}$ H. Gropengiesser, NASA TT-F-12, 786, 1970.

${ }^{15}$ T. L. Jackson and C. E. Grosch, ICASE Report No. 88-33, 1988.

${ }^{16} \mathrm{~N}$. Sandham and W. Reynolds, AIAA Paper No. 89-0371, 1989.

${ }^{17}$ C. K. W. Tam and F. Q. Hu, J. Fluid Mech. 203, 51 (1989).

${ }^{18}$ P. E. Dimotakis, AIAA Paper No. 89-0262, 1989. 